\title{
Gold ion parameters for the 2015 PP-on-Au setup in RHIC
}

\author{
C. J. Gardner
}

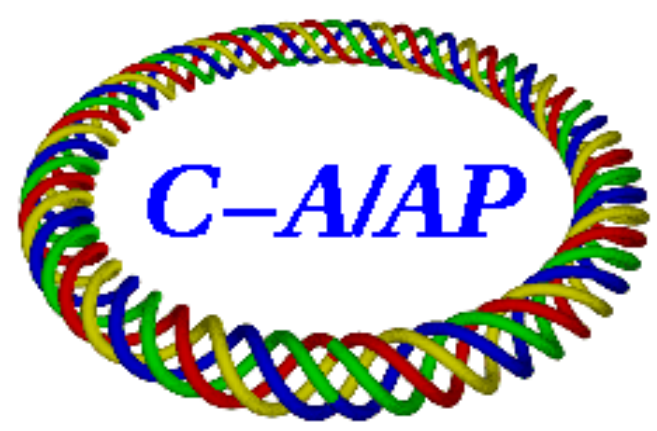

\section{Collider-Accelerator Department Brookhaven National Laboratory Upton, NY 11973}

\section{U.S. Department of Energy Office of Science, Office of Nuclear Physics}

Notice: This document has been authorized by employees of Brookhaven Science Associates, LLC under Contract No. DE-SC0012704 with the U.S. Department of Energy. The United States Government retains a nonexclusive, paid-up, irrevocable, world-wide license to publish or reproduce the published form of this document, or allow others to do so, for United States Government purposes. 


\title{
Gold Ion Parameters for the 2015 PP-on-Au Setup in RHIC
}

\author{
C.J. Gardner
}

August 25, 2015

In this note the nominal parameters for gold ions in Booster, AGS, and RHIC are given for the 2015 PP-on-Au setup in RHIC.

The setup parameters are summarized in Sections 17, 18, 19.

\section{Mass}

A gold ion with charge $e Q$ has $N=118$ neutrons, $Z=79$ protons, and $(Z-Q)$ electrons. Here $Q$ is an integer and $e$ is the positive elementary charge. The mass number is

$$
A=N+Z=197 \text {. }
$$

This is also called the number of nucleons. The mass energy equivalent of the ion is

$$
m c^{2}=a m_{u} c^{2}-Q m_{e} c^{2}+E_{Q}
$$

where $[1,2]$

$$
a=196.9665687(6)
$$

is the relative atomic mass of the neutral gold atom,

$$
m_{u} c^{2}=931.494061(21) \mathrm{MeV}
$$

is the mass energy equivalent of the atomic mass constant, and

$$
m_{e} c^{2}=0.510998928(11) \mathrm{MeV}
$$

is the electron mass energy equivalent. The binding energy $E_{Q}$ is the energy required to remove $Q$ electrons from the neutral gold atom. This 
amounts to $[3,4] 0.3324 \mathrm{MeV}$ for the helium-like gold ion $(Q=77)$ and $0.5170 \mathrm{MeV}$ for the fully stripped ion. For $Q=32$ we have $E_{Q}=14.5 \mathrm{KeV}$. Thus the mass energy equivalents for the Au32+, Au77+, and Au79+ ions are

$$
\begin{aligned}
& m c^{2}(\mathrm{Au} 32+)=183.456851494 \mathrm{GeV} \\
& m c^{2}(\mathrm{Au} 77+)=183.434174442 \mathrm{GeV}
\end{aligned}
$$

and

$$
m c^{2}(\mathrm{Au} 79+)=183.433337044 \mathrm{GeV} .
$$

\section{Kinetic Parameters}

In a circular accelerator the ion moves along an orbit of circumference $C$ with revolution frequency $f$. The radius of the orbit is defined to be $R=C /(2 \pi)$. The velocity of the ion is then

$$
v=2 \pi R f .
$$

This gives momentum, energy, and kinetic energy

$$
p=m c \beta \gamma, \quad E=m c^{2} \gamma, \quad W=m c^{2}(\gamma-1)
$$

where

$$
\beta=v / c, \quad \gamma=1 / \sqrt{1-\beta^{2}} .
$$

The magnetic rigidity of the ion in units of $\mathrm{Tm}$ is

$$
B \rho=k c p / Q
$$

where $k=10^{9} / 299792458$ and $c p$ is given in units of $\mathrm{GeV}$. The angular frequency is

$$
\omega=2 \pi f .
$$

We also define the phase-slip factor

$$
\eta=\frac{1}{\gamma_{t}^{2}}-\frac{1}{\gamma^{2}}
$$

where $\gamma_{t}$ is the transition gamma. Note that as defined here, $\eta$ is negative below transition and positive above transition. 


\section{RF Parameters}

1. The stationary bucket area is

$$
A_{S}=8 \frac{R_{s}}{h c}\left\{\frac{2 e Q V_{g} E_{s}}{\pi h\left|\eta_{s}\right|}\right\}^{1 / 2}
$$

where $h$ is the RF harmonic number, $V_{g}$ is the total RF gap voltage per turn, and the subscript " $s$ " denotes parameter values for the synchronous particle.

2. The half-height of a bucket is

$$
\Delta E=\left(\frac{h \omega_{s}}{8 \sqrt{2}}\right) A_{S}\left|\left(\pi-2 \phi_{s}\right) \sin \phi_{s}-2 \cos \phi_{s}\right|^{1 / 2}
$$

where $\phi_{s}$ is the synchronous phase.

3. The synchronous phase is given by

$$
V_{g} \sin \phi_{s}=2 \pi R_{s} \rho_{s} \dot{B} / c
$$

where $\rho_{s}$ is the radius of curvature, $B$ is the magnetic field and $\dot{B}=d B / d t$. Employing Gaussian units $\left(R_{s}\right.$ and $\rho_{s}$ in $\mathrm{cm}$, $c=2.99792458 \times 10^{10} \mathrm{~cm} / \mathrm{s}$, and $\dot{B}$ in $\left.\mathrm{G} / \mathrm{s}\right)$ gives $V_{g} \sin \phi_{s}$ in Statvolts. Multiplying by 299.792458 then gives $V_{g} \sin \phi_{s}$ in Volts.

4. The width of a bucket is

$$
\Delta t=\frac{\left|\pi-\phi_{s}-\phi_{e}\right|}{h \omega_{s}}
$$

where the phase $\phi_{e}$ satisfies

$$
\cos \left(\pi-\phi_{s}\right)-\cos \phi_{e}=-\left(\pi-\phi_{s}-\phi_{e}\right) \sin \phi_{s} .
$$

5. The area of a bucket is

$$
A_{\mathrm{bk}}=\alpha\left(\phi_{s}\right) A_{S}
$$

where

$$
\alpha\left(\phi_{s}\right)=\frac{\sqrt{2}}{8} \int_{\phi_{L}}^{\phi_{R}}\left|\left(\pi-\phi_{s}-\phi\right) \sin \phi_{s}-\cos \phi_{s}-\cos \phi\right|^{1 / 2} d \phi .
$$


Below transition we have $\phi_{e}<\pi-\phi_{s}$ and the limits of integration are $\phi_{L}=\phi_{e}$ and $\phi_{R}=\pi-\phi_{s}$. Above transition we have $\pi-\phi_{s}<\phi_{e}$ and the limits of integration are $\phi_{L}=\pi-\phi_{s}$ and $\phi_{R}=\phi_{e}$. The integral $\alpha\left(\phi_{s}\right)$ must be evaluated numerically. An approximate expression is [5]

$$
\alpha\left(\phi_{s}\right) \approx \frac{1-\sin \phi_{s}}{1+\sin \phi_{s}}
$$

6. The synchrotron frequency for small-amplitude oscillations about $\phi_{s}$ is

$$
F_{s}=\frac{c}{2 \pi R_{s}}\left\{\frac{-h \eta_{s} e Q V_{g} \cos \phi_{s}}{2 \pi E_{s}}\right\}^{1 / 2}
$$

and the corresponding synchrotron tune is $Q_{s}=2 \pi F_{s} / \omega_{s}$. Note that measurement of $F_{s}$ gives a value for $V_{g} \cos \phi_{s}$, while measurement of $d B / d t$ gives a value for $V_{g} \sin \phi_{s}$. These two can be used to obtain $V_{g}$ and $\phi_{s}$.

7. Let $\phi_{l}$ and $\phi_{r}$ be the phases at the left and right boundries of a bunch matched to a bucket. We have

$$
\phi_{l}<\phi_{s}<\phi_{r}
$$

and the width of the bunch is

$$
\Delta t=\frac{\Delta \phi}{h \omega_{s}}, \quad \Delta \phi=\phi_{r}-\phi_{l} .
$$

In terms of $\Delta \phi$ and $\phi_{s}$ we have

$$
\phi_{r}=\frac{\Delta \phi}{2}+\arcsin \left\{\frac{\Delta \phi \sin \phi_{s}}{2 \sin (\Delta \phi / 2)}\right\}
$$

and

$$
\phi_{l}=-\frac{\Delta \phi}{2}+\arcsin \left\{\frac{\Delta \phi \sin \phi_{s}}{2 \sin (\Delta \phi / 2)}\right\} .
$$

If $\Delta \phi$ is small we have

$$
\sin (\Delta \phi / 2) \approx \frac{\Delta \phi}{2}, \quad \frac{\Delta \phi \sin \phi_{s}}{2 \sin (\Delta \phi / 2)} \approx \sin \phi_{s}
$$

and

$$
\phi_{l} \approx \phi_{s}-\frac{\Delta \phi}{2}, \quad \phi_{r} \approx \phi_{s}+\frac{\Delta \phi}{2} .
$$


8. The half-height of a bunch matched to a bucket is

$$
\Delta E=\left(\frac{h \omega_{s}}{8 \sqrt{2}}\right) A_{S}\left|\cos \phi_{r}-\cos \phi_{s}+\left(\phi_{r}-\phi_{s}\right) \sin \phi_{s}\right|^{1 / 2} .
$$

9. The area of a bunch matched to a bucket is

$$
A_{\mathrm{b}}=F\left(\phi_{s}, \Delta \phi\right) A_{S}
$$

where

$$
F\left(\phi_{s}, \Delta \phi\right)=\frac{\sqrt{2}}{8} \int_{\phi_{l}}^{\phi_{r}}\left|\cos \phi_{l}-\cos \phi+\left(\phi_{l}-\phi\right) \sin \phi_{s}\right|^{1 / 2} d \phi .
$$

The integral $F\left(\phi_{s}, \Delta \phi\right)$ must be evaluated numerically. If $\Delta \phi$ is small we have

$$
F\left(\phi_{s}, \Delta \phi\right) \approx \frac{\pi}{64}(\Delta \phi)^{2}\left|\cos \phi_{s}\right|^{1 / 2} .
$$

\section{Ring Parameters}

\begin{tabular}{|c|c|c|c|c|}
\hline Parameter & Booster & AGS & RHIC & Unit \\
\hline$C_{I}$ & $C_{b}$ & $C_{a}$ & $C_{r}+\delta C$ & $\mathrm{~m}$ \\
\hline$C_{E}$ & $C_{a} / 4$ & $4\left(C_{r}+\delta C\right) / 19$ & $C_{r}+\delta C$ & $\mathrm{~m}$ \\
\hline$\rho$ & 13.8656 & 85.378351 & 242.7806 & $\mathrm{~m}$ \\
\hline$\gamma_{\mathrm{tr}}$ & 4.832 & 8.5 & 22.89 & \\
\hline
\end{tabular}

Here $C_{I}$ and $C_{E}$ are the circumferences of the closed orbits in the machines at injection and extraction (or store) respectively. $C_{b}, C_{a}$, and $C_{r}$ are the circumferences of the "design" orbits in Booster, AGS, and RHIC respectively. These are

$$
C_{b}=201.780, \quad C_{a}=2 \pi(128.4526), \quad C_{r}=3833.845181
$$

meters. $\delta C$ is the shift (if any) of the RHIC orbit circumference from the design value $C_{r}$. Note that $4\left(C_{r} / 19\right)=2 \pi(128.4580) \mathrm{m}$ which gives an AGS radius at extraction approximately $5 \mathrm{~mm}$ larger than the "design" AGS radius $(128.4526 \mathrm{~m}$ ) reported by Bleser [6, 7]. The radius of curvature $\rho$ in the Booster and AGS main dipoles is given in Refs. [6, 7, 8]. The RHIC ring parameters are taken from Ref. [9] and from MAD runs by Steve Tepikian. 


\section{$5 \quad$ Initial Conditions and Assumptions}

1. The revolution frequency of the Au32+ ion (from EBIS) at Booster injection is $96.640 \mathrm{kHz}$. The radius is taken to be the nominal radius $C_{b} /(2 \pi)$.

2. The revolution frequency of the $\mathrm{Au} 32+$ ion at Booster extraction is $f=658.91 \mathrm{KHz}$ [10]. The radius is taken to be one fourth the nominal AGS radius $C_{a} /(2 \pi)$. The corresponding magnetic rigidity is $B \rho=9.4620277 \mathrm{Tm}$. The rigidity that can be extracted from Booster into the BTA line is limited by the F3 extraction kicker. The advertised limit is $B \rho=9.5 \mathrm{Tm}$ [11].

3. The set revolution frequency of the Au77+ ion at AGS injection is $f=163.125 \mathrm{KHz}$. This gives an energy loss of $2.453 \mathrm{MeV}$ per nucleon in the BTA stripper.

4. The magnetic rigidity of the Au79+ ion at RHIC injection is taken to be $B \rho=81.11378003 \mathrm{Tm}$.

5. The circumference shift in RHIC yellow ring at Au79+ injection is $\delta C=-3.7203 \mathrm{~mm}[12]$.

6. The circumference shift in RHIC yellow ring at PP injection is $\delta C=-6.24572 \mathrm{~mm}[12]$.

7. The circumference shift in RHIC yellow ring at Store is $\delta C=-6.14315 \mathrm{~mm}[12]$.

8. The magnetic rigidity of the Au79+ ion in RHIC at PP injection is $B \rho=195.647211941 \mathrm{Tm}[12]$.

9. The magnetic rigidity of the Au79+ ion at RHIC store is $B \rho=812.990761749 \mathrm{Tm}[12]$.

The parameter values given in the following sections are calculated with these initial conditions and assumptions. For many of the parameters more digits are given than would be warranted by the precision with which the parameter could be measured; this is done for computational convenience. 


\section{Bunch Merging}

The desired number of ions per bunch in RHIC is achieved by merging bunches in both Booster and AGS as described in $[13,14]$. Each of the merges is a 2 to 1 merge in which 2 adjacent bunches are merged into 1 . If the merge is done sufficiently slowly, the gross emittance of the merged bunch will be the sum of the emittances of the initial 2 bunches. In this case we say that the gross emittance has been conserved. If the merge is done too quickly, the merged bunch will be diluted with empty phase space, making its gross emittance larger than that of the slowly merged bunch. In this case we say that there has been emittance growth (even though the area of phase space occupied by beam has not changed). Careful measurements [15] by K.L. Zeno have shown that the growth in longitudinal emittance during the merges is relatively small.

\section{Longitudinal Emittance}

The longitudinal emittance per nucleon of unbunched beam in Booster at injection is

$$
\mathcal{E}=\frac{2}{A} \Delta E \Delta T
$$

where $\Delta E$ is the energy half-width of the beam,

$$
\Delta T=\frac{1}{f}=\frac{2 \pi R}{c \beta}
$$

is the revolution period, and $A$ is the number of nucleons. Using the differential relation

$$
\Delta E=\beta^{2} \frac{\Delta p}{p} m c^{2} \gamma
$$

we have

$$
\mathcal{E}=\frac{2 \beta^{2} \gamma}{f} \frac{m c^{2}}{A} \frac{\Delta p}{p}
$$

where $\Delta p$ is the momentum half-width of the unbunched beam. Taking

$$
f=96.640 \mathrm{kHz}
$$

gives

$$
\Delta T=10.3476821192 \mu \mathrm{s}
$$




$$
\beta=0.0650450626079, \quad \gamma=1.00212216641
$$

and

$$
\frac{2 \beta^{2} \gamma}{f}=87.7450074295 \mathrm{~ns}
$$

For Au32+ ions we have

$$
\frac{m c^{2}}{A}=0.931253053269 \mathrm{GeV}
$$

which gives

$$
\frac{2 \beta^{2} \gamma}{f} \frac{m c^{2}}{A}=81.7128060778 \mathrm{eV} \mathrm{s} .
$$

For a fractional momentum half-width of

$$
\frac{\Delta p}{p}=0.001
$$

equation (38) then gives longitudinal emittance (per nucleon)

$$
\mathcal{E}=0.0817128060778 \mathrm{eV} \mathrm{s} .
$$

For the merging scheme described in $[13,14]$, four Booster loads end up in one AGS bunch. The minimum longitudinal emittance of that bunch would then be

$$
4 \mathcal{E}=0.32685 \mathrm{eV} \mathrm{s} .
$$

This is to be compared with the measured value of $0.428 \mathrm{eV} \mathrm{s}$ obtained by Zeno [15].

Measurements $[15,16]$ show that the longitudinal emittance of unbunched Au32+ beam in Booster at injection can be made as small as

$$
\mathcal{E}=0.032 \mathrm{eV} \mathrm{s} .
$$

Unfortunately, this only can be done by increasing the RF capture time well beyond the $8 \mathrm{~ms}$ allowed for the nominal magnetic cycle. The corresponding fractional momentum half-width is

$$
\frac{\Delta p}{p}=0.0003916
$$

A detailed account of the longitudinal emittance evolution in Booster and AGS is given in [15] and [13]. 


\section{Minimum RF Voltage Required to Capture the Unbunched Beam}

In order to capture the unbunched beam into $h$ buckets we must have RF voltage $V_{g}$ (i.e. total gap voltage per turn) such that

$$
\mathcal{E} \leq \frac{h A_{S}}{A}
$$

where $A_{S}$ is given by (15). Thus we must have

$$
\frac{2 \beta^{2} \gamma}{f} \frac{m c^{2}}{A} \frac{\Delta p}{p} \leq \frac{8 R}{c A}\left\{\frac{2 e Q V_{g} E}{\pi h|\eta|}\right\}^{1 / 2}
$$

which gives

$$
\begin{gathered}
2 \beta^{2} \gamma\left(\frac{2 \pi R}{c \beta}\right) \frac{m c^{2}}{A} \frac{\Delta p}{p} \leq \frac{8 R}{c}\left(\frac{2 \gamma}{\pi h|\eta|}\right)^{1 / 2} \frac{m c^{2}}{A}\left(\frac{e Q V_{g}}{m c^{2}}\right)^{1 / 2} \\
\beta^{2} \gamma\left(\frac{\pi}{\beta}\right) \frac{\Delta p}{p} \leq 2\left(\frac{2 \gamma}{\pi h|\eta|}\right)^{1 / 2}\left(\frac{e Q V_{g}}{m c^{2}}\right)^{1 / 2} \\
\beta^{2} \gamma^{2} \pi^{2}\left(\frac{\Delta p}{p}\right)^{2} \leq\left(\frac{8 \gamma}{\pi h|\eta|}\right)\left(\frac{Q}{m c^{2}}\right) e V_{g}
\end{gathered}
$$

and

$$
\frac{1}{8} h \pi^{3} \beta^{2} \gamma|\eta|\left(\frac{m c^{2}}{Q}\right)\left(\frac{\Delta p}{p}\right)^{2} \leq e V_{g} .
$$

Here

$$
h=4
$$

and taking revolution frequency

$$
f=96.640 \mathrm{kHz}
$$

we have

$$
\eta=-0.952939329734
$$

and

$$
\frac{1}{8} h \pi^{3} \beta^{2} \gamma|\eta|=0.0626374709945 .
$$


For the Au32+ ion we have mass energy equivalent per unit charge

$$
\frac{m c^{2}}{Q}=5.73302660918 \mathrm{GeV}
$$

Taking fractional momentum half-width

$$
\frac{\Delta p}{p}=0.001
$$

then gives

$$
359.102287944 \text { volts } \leq V_{g}
$$

\section{Inflector Voltage}

At Booster injection, the voltage $V_{I}$ required for particles with mass $m$, velocity $c \beta$, and charge $e Q$ to follow the nominal trajectory through the inflector is given by

$$
e V_{I}=\frac{G}{R_{I}}\left(\frac{m c^{2}}{Q}\right) \beta^{2} \gamma
$$

Here $G=0.021 \mathrm{~m}$ is the gap between the cathode and septum of the inflector and $R_{I}=8.74123 \mathrm{~m}$ is the radius of curvature along the nominal trajectory. Using the values of $\beta, \gamma$, and $m c^{2} / Q$ given by (41) and (60), we obtain

$$
V_{I}=58.396 \mathrm{kV}
$$

for Au32+ ions from EBIS. Because of an unresolved calibration problem, the actual setpoint for the inflector voltage needs to be

$$
V_{I}(\text { setpoint })=59.740 \mathrm{kV} \text {. }
$$




\section{Booster Injection Field}

The nominal magnetic field in the Booster dipoles at injection is

$$
B=(B \rho) / \rho
$$

where $B \rho$ is given by (12) and $\rho$ is the nominal radius of curvature. Writing

$$
B \rho=\frac{10^{9}}{c}\left(\frac{m c^{2}}{Q}\right) \beta \gamma
$$

and using the values of $\beta, \gamma$, and $m c^{2} / Q$ given by (41) and (60), we obtain

$$
B \rho=1.24651715338 \mathrm{Tm} \text {. }
$$

Here we have used the mass energy equivalent $m c^{2}$ in units of $\mathrm{GeV}$ and the velocity of light in units of $\mathrm{m} / \mathrm{s}$. Using

$$
\rho=13.8656 \mathrm{~m}
$$

we then obtain

$$
B=898.999793284 \text { Gauss }
$$

for $\mathrm{Au} 32+$ ions from EBIS.

The magnetic field is measured with a Hall probe and the Booster Gauss Clock. The Hall probe sits in the reference dipole and gives the value of the field at BT0. The Gauss Clock gives the change in field between BT0 and the time of measurement. The measured field is defined to be the field at BT0 plus the field change given by the Gauss Clock. For Au32+ ions from EBIS the measured field at injection is

$$
B(\text { measured })=894.0 \text { Gauss. }
$$

\section{AGS Injection Field}

Similarly, the nominal magnetic field in the AGS dipoles at injection is $B=454.96$ Gauss for the Au77+ ions. The measured magnetic field is 482.0 gauss [17]. 


\section{BTA Stripper}

The stripper $[26,27]$ used to strip gold ions in the BTA (Booster-To-AGS) transfer line consists of a $6.45 \mathrm{mg} / \mathrm{cm}^{2}$ aluminum foil followed by a 8.39 $\mathrm{mg} / \mathrm{cm}^{2}$ carbon foil. In Section 21 we use these surface densities to calculate the energy loss of Au77+ ions in the foils.

\section{AGS Injection Septum Magnet Current}

The field required in the L20 septum magnet is

$$
B=(B \rho) / \rho
$$

where $B \rho$ is the magnetic rigidity of the beam and $\rho=18.625 \mathrm{~m}$ [19] is the radius of curvature of the nominal trajectory through the magnet. The required current is given by

$$
N I=g B / \mu_{0}
$$

where $N=1$ is the number of conductor turns; $g=0.0467 \mathrm{~m}$ [19] is the magnet gap; and $\mu_{0}=4 \pi \times 10^{-7} \mathrm{Tm} / \mathrm{A}$.

For Au77+ ions at injection, the magnetic rigidity is $B \rho=3.88434088 \mathrm{Tm}$. This gives $B=0.208555 \mathrm{~T}$ and $I=7750 \mathrm{~A}$.

For comparison, the magnetic rigidity of polarized protons at AGS injection is $B \rho=7.205178 \mathrm{Tm}$. This gives $B=0.3869 \mathrm{~T}$ and $I=14380 \mathrm{~A}$.

\section{AGS Injection Kicker Current}

The current required in the A5 kicker is $[18,19]$

$$
I=\frac{B \rho}{K} \sin \phi
$$

where

$$
K=1.8718 \times 10^{-5} \mathrm{Tm} / \mathrm{A}
$$

and

$$
\phi=3.35 \text { milliradians }
$$

is the desired kick angle. Using the calculated values of $B \rho$ at AGS injection we obtain a current of $695.2 \mathrm{~A}$ for Au77+ ions. The maximum available current is $1100 \mathrm{~A}$. 


\section{AGS Injection Kicker Short Pulse Waveforms}

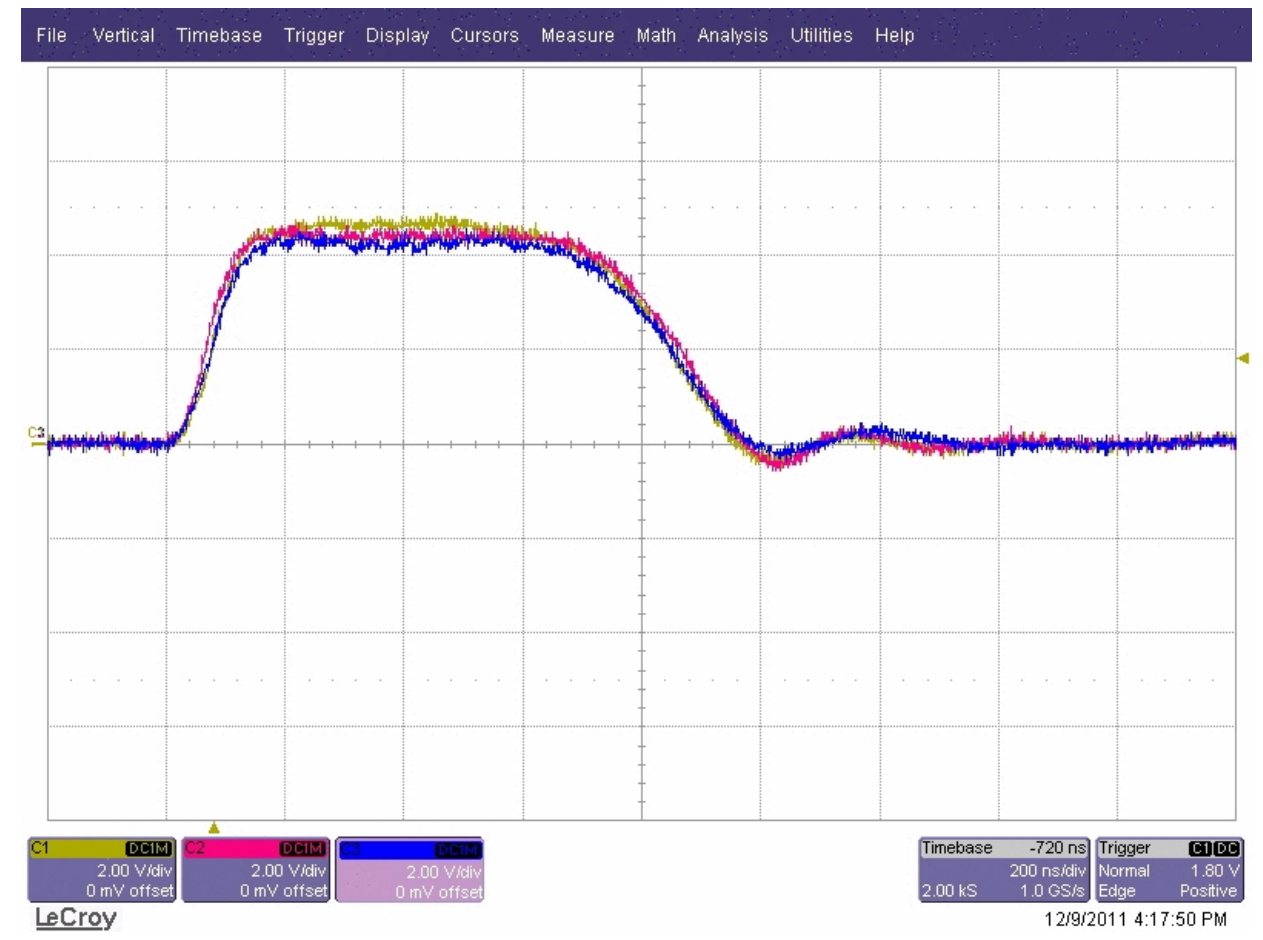

Figure 1: AGS injection kicker waveforms in the short pulse mode. The three traces are from the three modules of the kicker. They were taken by Yugang Tan on 9 Dec 2011. The time per division is 200 ns. The RF bucket width on the AGS injection porch is $383 \mathrm{~ns}$ for Au77+ ions. In order to put beam into adjacent buckets, the rise time of the kicker must be less than or equal to $T-W$, where $T$ is the bucket width and $W$ the bunch width. The rise time is approximately $100 \mathrm{~ns}$, which implies that the bunch width must be less than or equal to 283 for Au77+ bunches. A single bunch of this width easily fits on the flattop portion of the pulse which is some 600 ns long. The total width of the pulse is approximately 1000 ns. With this kicker pulse one could in principle fill 14 of the 16 RF buckets on the AGS injection porch. The pulse is too wide to fill the remaining buckets without interfering with beam in the adjacent buckets. This is not an issue as only 8 of the buckets need to be filled. One workable filling pattern is four adjacent filled buckets followed by four adjacent empty buckets, followed by another four adjacent filled buckets. Another workable pattern is eight adjacent filled buckets followed eight empty buckets. 


\section{AGS Injection Kicker Long Pulse Waveforms}

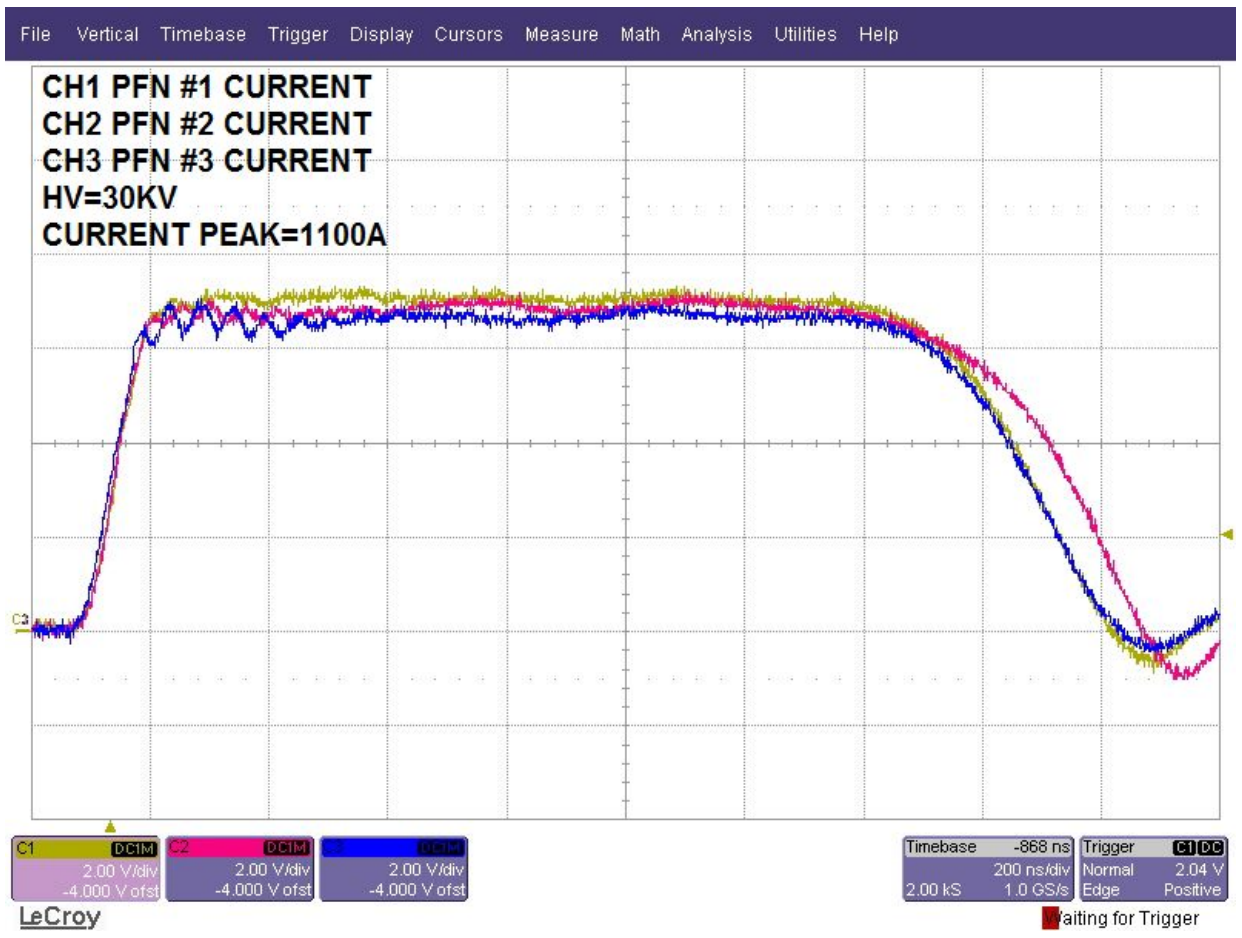

Figure 2: AGS injection kicker waveforms in the long pulse mode. The three traces are from the three modules of the kicker. They were taken by Yugang Tan in Oct 2010. The time per division is $200 \mathrm{~ns}$. The RF bucket width on the AGS injection porch is 383 ns for Au77+ ions. Here the flattop portion of the pulse is some $1300 \mathrm{~ns}$ long. The total pulse width is approximately 2000 ns. In principle this kicker pulse could be used to fill 8 buckets wtih a filling pattern of four adjacent filled buckets followed by four adjacent empty buckets, followed by another four adjacent filled buckets. 


\section{Gold in Booster}

\begin{tabular}{|c|c|c|c|c|}
\hline Parameter & Injection & Merge porch & Extraction & Unit \\
\hline$Q$ & 32 & 32 & 32 & \\
\hline$m c^{2}$ & 183.456851 & 183.456851 & 183.456851 & $\mathrm{GeV}$ \\
\hline$W / A$ & 1.9762739452 & 49.259795 & 107.75879 & $\mathrm{MeV}$ \\
\hline$c p / A$ & 60.701960016 & 306.87652 & 460.77475 & $\mathrm{MeV}$ \\
\hline$E / A$ & 0.9332293272 & 0.98051285 & 1.0390118 & $\mathrm{GeV}$ \\
\hline$B \rho$ & 1.24651715338 & 6.3017214 & 9.4620277 & $\mathrm{Tm}$ \\
\hline$\beta$ & 0.065045062608 & 0.31297552 & 0.44347401 & \\
\hline$\gamma-1$ & 0.002122166406 & 0.052896251 & 0.11571376 & \\
\hline$\eta$ & -0.953 & -0.859 & -0.7605 & \\
\hline$\epsilon_{H}(95 \%)$ & $12.1 \pi$ & $12.1 \pi$ & $12.1 \pi$ & $\mathrm{mm} \mathrm{mrad}$ \\
\hline$\epsilon_{V}(95 \%)$ & $5.68 \pi$ & $5.68 \pi$ & $5.68 \pi$ & $\mathrm{mm} \mathrm{mrad}$ \\
\hline$h$ & 4 & 1 & 1 & \\
\hline$h f$ & 386.560 & 465.000 & 658.910 & $\mathrm{KHz}$ \\
\hline$R$ & $201.780 /(2 \pi)$ & $201.780 /(2 \pi)$ & $128.4526 / 4$ & $\mathrm{~m}$ \\
\hline
\end{tabular}

Here $\epsilon_{H}$ and $\epsilon_{V}$ are the normalized horizontal and vertical transverse emittances. These follow from the assumption that during injection the horizontal and vertical acceptances in Booster are completely filled. The horizontal and vertical acceptances are $185 \pi$ and $87 \pi \mathrm{mm} \mathrm{mrad}$ (un-normalized) respectively.

\begin{tabular}{|c|c|c|c|c|c|c|}
\hline Parm & Injection & Injection & Ext & Ext & Ext & Unit \\
\hline$V_{g}$ & 0.403 & 5.730 & 25.2 & 25.2 & 25.2 & $\mathrm{kV}$ \\
$A_{S}$ & 4.263 & 16.076 & 318.54 & 318.54 & 318.54 & $\mathrm{eV} \mathrm{s}$ \\
$d B / d t$ & 0 & 0 & $\mathbf{7 0}$ & $\mathbf{3 5}$ & $\mathbf{0}$ & $\mathrm{G} / \mathrm{ms}$ \\
\hline$\phi_{s}$ & 0 & 0 & 50.999 & 22.866 & 0 & $\mathrm{deg}$ \\
$F_{s}$ & 0.3065 & 1.1557 & 0.8139 & 0.9849 & 1.0260 & $\mathrm{kHz}$ \\
$A_{\mathrm{bk}}$ & 4.263 & 16.076 & 36.294 & 140.88 & 318.54 & $\mathrm{eV} \mathrm{s}$ \\
\hline$A_{b}$ & 1.576 & 3.8907 & 17.533 & 17.533 & 17.533 & $\mathrm{eV} \mathrm{s}$ \\
$\Delta t$ & 1196 & 947.0 & 299.3 & 263.8 & 257.7 & $\mathrm{~ns}$ \\
$\Delta E$ & 0.8596 & 2.654 & 37.98 & 42.50 & 43.45 & $\mathrm{MeV}$ \\
\hline
\end{tabular}

\begin{tabular}{|c|c|c|c|c|}
\hline Parameter & Injection & Injection & Extraction & Unit \\
\hline No. Bunches & 4 & 4 & 1 & \\
\hline Bucket Width & 2586.92053 & 2586.92053 & 1517.65795 & ns \\
\hline Ions/Bunch & $1.12 / 4$ & $1.12 / 4$ & 1.007 & $10^{9}[20]$ \\
\hline Bunch Area & $0.032 / 4[16]$ & $0.0790 / 4[21]$ & $0.089[22]$ & $\mathrm{eV} \mathrm{s} / A$ \\
\hline
\end{tabular}




\section{Gold in AGS}

\begin{tabular}{|c|c|c|c|c|}
\hline Parameter & Injection & Transition & Extraction & Unit \\
\hline$Q$ & 77 & 77 & 77 & \\
\hline$m c^{2}$ & 183.434174 & 183.434174 & 183.434174 & $\mathrm{GeV}$ \\
\hline$W / A$ & 0.10529199 & 6.98353456 & 8.86486803967 & $\mathrm{GeV}$ \\
\hline$c p / A$ & 0.45515837 & 7.85970883 & 9.75165192744 & $\mathrm{GeV}$ \\
\hline$E / A$ & 1.0364299 & 7.91467250 & 9.79600598100 & $\mathrm{GeV}$ \\
\hline$B \rho$ & 3.88434088 & 67.0750887 & 83.2210113659 & $\mathrm{Tm}$ \\
\hline$\beta$ & 0.43915981 & 0.993055472 & 0.995472230862 & \\
\hline$\gamma$ & 1.1130788 & 8.5000 & 10.5204669965 & \\
\hline$\eta$ & -0.793 & 0.0 & 0.00481 & \\
\hline$\epsilon_{H}(95 \%)$ & $\leq 12 \pi$ & $\leq 12 \pi$ & $\leq 12 \pi$ & $\mathrm{mm} \mathrm{mrad}$ \\
\hline$\epsilon_{V}(95 \%)$ & $\leq 12 \pi$ & $\leq 12 \pi$ & $\leq 12 \pi$ & $\mathrm{mm} \mathrm{mrad}$ \\
\hline$h$ & 16 & 12 & 12 & \\
\hline$h f$ & 2.610000 & 4.42642072 & 4.43701154342 & $\mathrm{MHz}$ \\
\hline$R$ & 128.4526 & 128.4526 & 128.457856737 & $\mathrm{~m}$ \\
\hline
\end{tabular}

\begin{tabular}{|c|c|c|c|c|}
\hline Parameter & Injection & Injection & Extraction & Unit \\
\hline$h$ & 16 & 4 & 12 & \\
\hline$V_{g}$ & 17.267 & 6.567 & 192.0 & $\mathrm{kV}$ \\
$A_{S}$ & 24.998 & 123.33 & 5070 & $\mathrm{eV} \mathrm{s}$ \\
$d B / d t$ & 0 & 0 & 0 & $\mathrm{G} / \mathrm{ms}$ \\
\hline$\phi_{s}$ & 0 & 0 & 180 & degrees \\
$F_{s}$ & 1.347 & 0.4154 & 0.0985 & $\mathrm{kHz}$ \\
$A_{\mathrm{bk}}$ & 24.998 & 123.33 & 5070 & $\mathrm{eV} \mathrm{s}$ \\
\hline$A_{b}$ & 19.7 & 84.316 & 137.9 & $\mathrm{eV} \mathrm{s}$ \\
$\Delta t$ & 287.0 & $1034[23]$ & 26.8 & $\mathrm{~ns}$ \\
$\Delta E$ & 47.31 & 55.13 & 3281 & $\mathrm{MeV}$ \\
\hline
\end{tabular}

\begin{tabular}{|c|c|c|c|c|}
\hline Parameter & Injection & Injection & Extraction & Unit \\
\hline$h$ & 16 & 4 & 12 & \\
\hline Bucket Width & 383.142 & 1532.567 & 225.377 & $\mathrm{~ns}$ \\
\hline No. of Bunches & 8 & 2 & 2 & \\
\hline Ions/Bunch & 0.55725 & 2.229 & 2.055 & $10^{9}[20]$ \\
\hline Bunch Area & $0.10[13]$ & $0.428[13]$ & $0.70[24]$ & $\mathrm{eV} \mathrm{s} / A$ \\
\hline
\end{tabular}




\section{Gold in RHIC}

\begin{tabular}{|c|c|c|c|c|}
\hline Parameter & Injection & PP Injection & Store & Unit \\
\hline$Q$ & 79 & 79 & 79 & \\
\hline$m c^{2}$ & 183.433337 & 183.433337 & 183.433337 & $\mathrm{GeV}$ \\
\hline$W / A$ & 8.86482757134 & 22.6082598553 & 96.8121411674 & $\mathrm{GeV}$ \\
\hline$c p / A$ & 9.75160741084 & 23.5209701874 & 97.7388396187 & $\mathrm{GeV}$ \\
\hline$E / A$ & 9.79596126192 & 23.5393935459 & 97.7432748580 & $\mathrm{GeV}$ \\
\hline$B \rho$ & 81.11378003 & 195.647211941 & 812.990761749 & $\mathrm{Tm}$ \\
\hline$\beta$ & 0.995472230863 & 0.999217339291 & 0.999954623586 & \\
\hline$\gamma$ & 10.5204669974 & 25.2803585393 & 104.972331951 & \\
\hline$\eta$ & -0.00713 & 0.0003439 & 0.001818 & \\
\hline$\epsilon_{H}(95 \%)$ & $\leq 10 \pi$ & $\leq 10 \pi$ & $\leq 10 \pi$ & $\mathrm{mm} \mathrm{mrad}$ \\
\hline$\epsilon_{V}(95 \%)$ & $\leq 10 \pi$ & $\leq 10 \pi$ & $\leq 10 \pi$ & $\mathrm{mm} \mathrm{mrad}$ \\
\hline$f$ & 77.8423077794 & 78.1352131060 & 78.1928640023 & $\mathrm{kHz}$ \\
\hline$h$ & 360 & 360 & 360 & \\
\hline$h f$ & 28.0232308006 & 28.1286767182 & 28.1494310408 & $\mathrm{MHz}$ \\
\hline$\delta C$ & -3.7203 & -6.24572 & -6.14315 & $\mathrm{~mm}$ \\
\hline
\end{tabular}

\begin{tabular}{|c|c|c|c|}
\hline Parameter & Injection & Store & Unit \\
\hline$h$ & 360 & 2520 & \\
\hline$V_{g}$ & 393.1 & 3000 & $\mathrm{kV}$ \\
$A_{S}$ & 174.4 & 162.7 & $\mathrm{eV} \mathrm{s}$ \\
$d B / d t$ & 0 & 0 & $\mathrm{G} / \mathrm{ms}$ \\
\hline$\phi_{s}$ & 0 & 180 & degrees \\
$F_{s}$ & 0.200 & 0.234 & $\mathrm{kHz}$ \\
$A_{\mathrm{bk}}$ & 174.4 & 162.7 & $\mathrm{eV} \mathrm{s}$ \\
$A_{b}$ & 137.9 & 137.9 & $\mathrm{eV} \mathrm{s}$ \\
\hline$A_{b}$ & 0.70 & 0.70 & $\mathrm{eV} \mathrm{s} / \mathrm{A}[25]$ \\
$\Delta t$ & 26.8 & 4.03 & $\mathrm{~ns}$ \\
$\Delta E$ & 3549 & 23900 & $\mathrm{MeV}$ \\
\hline
\end{tabular}




\section{Center-of-Mass Energy for Proton-Ion Collisions in RHIC}

Let $E_{1}$ and $P_{1}$ be the energy and momentum of an ion circulating in RHIC, and let $E_{2}$ and $-P_{2}$ be the energy and momentum of the counter-circulating ion. The counter-circulating ion may be identical to the circulating one or it may be some other kind of ion.

The center-of-mass (CM) mass-energy equivalent, $M c^{2}$, is given by the Lorentz invariant

$$
M^{2} c^{4}=\left(E_{1}+E_{2}\right)^{2}-\left(c P_{1}-c P_{2}\right)^{2}
$$

where

$$
E_{1}=m_{1} c^{2} \gamma_{1}, \quad c P_{1}=m_{1} c^{2} \beta_{1} \gamma_{1}
$$

and

$$
E_{2}=m_{2} c^{2} \gamma_{2}, \quad c P_{2}=m_{2} c^{2} \beta_{2} \gamma_{2}
$$

Thus we have

$$
\begin{gathered}
M^{2}=\left(m_{1} \gamma_{1}+m_{2} \gamma_{2}\right)^{2}-\left(m_{1} \beta_{1} \gamma_{1}-m_{2} \beta_{2} \gamma_{2}\right)^{2} \\
M^{2}=m_{1}^{2}\left(\gamma_{1}^{2}-\beta_{1}^{2} \gamma_{1}^{2}\right)+m_{2}^{2}\left(\gamma_{2}^{2}-\beta_{2}^{2} \gamma_{2}^{2}\right)+2 m_{1} m_{2} \gamma_{1} \gamma_{2}\left(1+\beta_{1} \beta_{2}\right)
\end{gathered}
$$

and

$$
M^{2}=m_{1}^{2}+m_{2}^{2}+2 m_{1} m_{2} \gamma_{1} \gamma_{2}\left(1+\beta_{1} \beta_{2}\right) .
$$

Here we have used the identities

$$
\gamma_{1}^{2}-\beta_{1}^{2} \gamma_{1}^{2}=1, \quad \gamma_{2}^{2}-\beta_{2}^{2} \gamma_{2}^{2}=1
$$

For Proton-Ion collisions we take

$$
\begin{aligned}
& \beta_{1}=\beta_{p}, \quad \gamma_{1}=\gamma_{p} \\
& \beta_{2}=\beta_{I}, \quad \gamma_{2}=\gamma_{I}
\end{aligned}
$$

and

$$
m_{1}=m_{p}, \quad m_{2}=m_{I} / A
$$

where $A$ is the atomic number (number of nucleons) of the ion, and the subscripts $p$ and $I$ refer to the proton and ion respectively. Thus (82) becomes

$$
M^{2}=m_{p}^{2}+\left(\frac{m_{I}}{A}\right)^{2}+2 m_{p}\left(\frac{m_{I}}{A}\right) \gamma_{p} \gamma_{I}\left(1+\beta_{p} \beta_{I}\right)
$$


where

$$
\beta_{p} \gamma_{p}=\left\{\gamma_{p}^{2}-1\right\}^{1 / 2}, \quad \beta_{I} \gamma_{I}=\left\{\gamma_{I}^{2}-1\right\}^{1 / 2}
$$

The mass-energy equivalent of the proton is [2]

$$
m_{p} c^{2}=0.938272046(21) \mathrm{GeV} .
$$

For the $\mathrm{Au} 79+$ ion, the atomic number is

$$
A=197
$$

and the mass-energy equivalent is

$$
m_{I} c^{2}=183.433337044 \mathrm{GeV} .
$$

For polarized protons, the desired values of $\gamma_{p}$ are quantized by the relation

$$
G \gamma_{p}=k+\frac{1}{2}
$$

where $k$ is a non-negative integer and

$$
G=\left(g_{p}-2\right) / 2 .
$$

Here the proton $\mathrm{g}$ factor is [2]

$$
g_{p}=5.585694713(46)
$$

which gives

$$
G=1.79284735650 .
$$

For polarized protons with

$$
k=198
$$

and Au79+ ions with

$$
\gamma_{I}=104.997926997
$$

we then have CM mass-energy equivalent

$$
M c^{2}=201.533112539 \mathrm{GeV} .
$$




\section{Au77+ Energy Loss in the BTA Stripper Foils}

The stripper used to strip gold ions consists of a $6.45 \mathrm{mg} / \mathrm{cm}^{2}$ aluminum foil followed by a $8.39 \mathrm{mg} / \mathrm{cm}^{2}$ "glassy" carbon foil $[26,27]$. We can estimate the energy loss in the foils as follows:

The kinetic energy of a proton that has the same velocity as the Au77+ ion just upstream of the aluminum foil is

$$
W_{p}=108.6 \mathrm{MeV} .
$$

The rate of energy loss of a proton passing through the foil with kinetic energy $W_{p}$ is [28]

$$
-\frac{d E_{p}}{d x}=5.348 \mathrm{MeV} \mathrm{cm}^{2} / \mathrm{g} .
$$

The rate of energy loss of the Au77+ ion is obtained by scaling the Bethe-Bloch result for protons [29]. Thus

$$
-\frac{d E}{d x}=-Z^{2} \frac{d E_{p}}{d x} \mathrm{~cm}^{2} / \mathrm{g}
$$

where $Z=77$. Multiplying this by the surface density of the aluminum foil $\left(6.45 \mathrm{mg} / \mathrm{cm}^{2}\right)$ gives

$$
\Delta E_{a}=1.038 \mathrm{MeV} \text { per nucleon. }
$$

This is the energy lost by the Au77+ ion upon passing through the aluminium foil. The kinetic energy of a proton that has the same velocity as the Au77+ ion just downstream of the aluminum foil is then

$$
W_{p}=107.5 \mathrm{MeV} \text {. }
$$

The rate of energy loss of a proton passing through the carbon foil with this kinetic energy is [28]

$$
-\frac{d E_{p}}{d x}=6.180 \mathrm{MeV} \mathrm{cm}^{2} / \mathrm{g} .
$$

Using this result in (101) with $Z=77$, and multiplying by the surface density of the carbon foil $\left(8.39 \mathrm{mg} / \mathrm{cm}^{2}\right)$ gives

$$
\Delta E_{c}=1.561 \mathrm{MeV} \text { per nucleon. }
$$

The total energy lost upon passing through both foils is then

$$
\Delta E=\Delta E_{a}+\Delta E_{c}=2.599 \mathrm{MeV} \text { per nucleon. }
$$

This agrees reasonably well with the value $2.453 \mathrm{MeV}$ per nucleon obtained in Section 5. 


\section{References}

[1] J.S. Coursey, D.J. Schwab, and R.A. Dragoset, "Atomic Weights and Isotopic Compositions", Nuclear Physics Data, Physical Reference Data, www.nist.gov.

[2] P.J. Mohr and B.N. Taylor, "Values of Fundamental Physical Constants", Physical Constants, Physical Reference Data, www.nist.gov.

[3] K.A. Brown, C. Gardner and P. Thieberger, "Rest Mass of Fully Stripped Ions in RHIC: Updated Values", C-A/AP/Note 293, October 2007.

[4] G.C. Rodrigues, P. Indelicato, J.P. Santos, P. Patte, and F. Parente, "Systematic Calculation of Total Atomic Energies of Ground State Configurations", Atomic Data and Nuclear Data Tables 86 (2004) $117-233$.

[5] S.Y. Lee, "Accelerator Physics", World Scientific, 1999, pp. 229-230

[6] E.J. Bleser, "Where are the AGS Magnets", Accelerator Division Technical Note 215, May 20, 1985.

[7] C.J. Gardner, "Notes on Orbit Equations in the AGS", C-A/AP/Note 164, September 2004.

[8] R. Thern, "Booster Dipole Production Measurements", Booster Technical Note 190, March 13, 1991.

[9] W. Fischer and S. Peggs, "RHIC Parameters", Revision of 3/18/97.

[10] K.L. Zeno, Booster-AGS-EBIS-2012 elog, 16 May 2012, entry 15:50.

[11] W. Zhang, R. Sanders, A. Soukas and J. Tuozzolo, "An Overview of the Fast Injection-Extraction Kicker Systems of the Brookhaven AGS-Booster Complex", PAC99, pp. 1264-1266.

[12] As calculated by Al Marusic. The circumference shifts are due to the unequal rigidities of polarized protons (PP) and Au79+ ions in the DX magnets.

[13] C.J. Gardner, et al, "Operation of the RHIC Injector Chain with Ions from EBIS", Proceedings of IPAC2015 
[14] C.J. Gardner, "Simulations of Merging and Squeezing Bunches in Booster and AGS", C-A/AP/Note 460, July 2012.

[15] K.L. Zeno, "Longitudinal Emittance Measurements in the Booster and AGS during the 2014 RHIC Gold Run", C-A/AP/Note 523, August 2014.

[16] K.L. Zeno, Booster-AGS-EBIS-2014 elog, 3 July 2014, entries 13:29 and 13:33.

[17] K.L. Zeno, Booster-AGS-EBIS-2014 elog, 21 February 2014, entry 19:23.

[18] C.J. Gardner, "Determination of the AGS Injection Kicker Strength from Beam Measurements", C-A/AP/Note 91, December 2002.

[19] C.J. Gardner, "AGS Injection with an Additional Kicker in the A10 Straight Section", C-A/AP/Note 217, September 2005.

[20] K.L. Zeno, Booster-AGS-EBIS-2014 elog, 29 May 2014, entry 18:18.

[21] K.L. Zeno, Booster-AGS-EBIS-2014 elog, 26 June 2014, entries 12:42 and 13:59.

[22] K.L. Zeno, Booster-AGS-EBIS-2014 elog, 4 June 2014, entry 17:48 and 24 June 2014, entry 17:27.

[23] K.L. Zeno, Booster-AGS-EBIS-2014 elog, 3 February 2014, entry $17: 40$.

[24] K.L. Zeno, Booster-AGS-EBIS-2014 elog, 21 March 2014, entry 18:13; 23 April 2014, entries 21:04; 2 May 2014, entries 17:40 and 17:57.

[25] Here we take the longitudinal emittance to be the same as that at AGS extraction. This gives a lower bound on the longitudinal emittance in RHIC.

[26] C.J. Gardner, et al, "Setup and Performance of the RHIC Injector Accelerators for the 2007 Run with Gold Ions", Proceedings of PAC07, pp. 1862-1864.

[27] P. Thieberger, et al, "Improved Gold Ion Stripping at 0.1 and 10 $\mathrm{GeV} /$ nucleon for the Relativistic Heavy Ion Collider", Phys. Rev. ST Accelerators and Beams 11, 011001 (2008). 
[28] M.J. Berger, J.S. Coursey, M.A. Zucker and J. Chang, "Stopping-Power and Range Tables for Electrons, Protons, and Helium Ions", www.nist.gov/physlab/data/star/index.cfm

[29] W.R. Leo, "Techniques for Nuclear and Particle Physics Experiments", Second Revised Edition, Springer-Verlag, 1994, pp. $24-28$. 